\title{
Nutritional values of selected tropical browse plants for commercial deer farming
}

\author{
ZA Idayu ', AS Haji Baba 1, KK Md Zain 2 \\ IIPT (2PAS), University of Malaya, 59100 Kuala Lumpur, Malaysia
}

Several species of deer are farmed in Malaysia including rusa deer (Cervus timorensis), fallow deer (Dama dama) and chital deer (Axis axis). The feeding system commonly in use is free grazing and browsing of plants available from secondary forest within the confinement. The nutritional values of many of these browse plants however are not fully known.

The present study has attempted to determine the nutritional value of the shoots and leaves of selected browse plants (triplicate sample/species, each from different location) found to be edible and highly palatable for deer kept in captivity throughout Peninsular Malaysia. The chemical composition of these feedstuffs was analysed using proximate analysis (AOAC, 1980, 13, 20) and detergent method (Goering and Van Soest, 1970, Dept Agric, 379, Washington DC). Metabolic energy content was determined by the gas test method (Menke et al, J Agric Sci, 93, 217-222). Mineral content was estimated by inductively coupled plasma (ICP) (Laid et al, 1991, Soil Science Soc Am J, 55 (1), 274-278).

The plants contained relatively high amount of water $(>67 \%$ ) with crude protein ranging from $24.3 \%$ to $35.6 \%$ DM. Metabolic energy (ME) contents ranged between 6.3 to $8.4 \mathrm{kcal} / \mathrm{kg}$ DM. Preliminary digestibility trials using the nylon bag technique in goats indicate that plant materials are moderately digestible $(\sim 60-70 \%)$. The present nutritional evaluation of plants indicates that, except for a few species, most of the edible tropical browse plants are suitable only for supplementary feeding. Large scale cultivation of a few browse plant species may be suitable and economical for feeding deer in commercial deer farming.

$\begin{array}{lcccccccccc}\text { Plant species } & \mathrm{H}_{2} \mathrm{O} & \mathrm{CP} \% & \mathrm{CF} \% & \mathrm{EE} \% & \text { Ash } & \text { NFE \% } & \mathrm{ME} & \mathrm{NDF} \% & \text { ADF \% Ca : P } \\ \text { Saudropus } & 75.8 & 30.1 & 9.6 & 8.2 & 10.0 & 42.3 & 7.6 & 24.2 & 18.5 & 0.66 \\ \text { androgynus } & \pm 0.9 & \pm 8.1 & \pm 0.2 & \pm 1.5 & \pm 3.7 & \pm 2.6 & \pm 0.1 & \pm 1.7 & \pm 0.5 & \\ \text { Gnetum } & 80.8 & 26.4 & 8.6 & 2.1 & 7.5 & 55.3 & 8.4 & 19.0 & 29.4 & 1.08 \\ \text { gnemum } & \pm 3.1 & \pm 0.8 & \pm 0.4 & \pm 0.4 & \pm 1.4 & \pm 3.9 & \pm 0.3 & \pm 0.6 & \pm 0.3 & \\ \text { Sapium } & 74.8 & 24.3 & 14.8 & 4.1 & 5.6 & 51.4 & 7.4 & 22.2 & 31.3 & 0.70 \\ \text { baccatum } & \pm 6.0 & \pm 1.9 & \pm 0.1 & \pm 0.4 & \pm 0.3 & \pm 1.9 & \pm 1.2 & \pm 3.0 & \pm 0.8 & \\ \text { Premna } & 81.3 & 26.8 & 12.0 & 4.1 & 9.1 & 47.9 & 7.3 & 40.8 & 45.9 & 1.14 \\ \text { cordifolia } & \pm 1.6 & \pm 2.2 & \pm 1.3 & \pm 0.8 & \pm 1.1 & \pm 3.1 & \pm 1.6 & \pm 3.2 & \pm 1.1 & \\ \text { Mikania } & 83.7 & 34.2 & 20.7 & 3.2 & 11.2 & 30.6 & 7.6 & 49.8 & 28.5 & 6.85 \\ \text { micrantha } & \pm 0.3 & \pm 1.9 & \pm 1.0 & \pm 0.1 & \pm 1.2 & \pm 2.3 & \pm 1.0 & \pm 2.3 & \pm 2.4 & \\ \text { Mussaenda } & 84.8 & 29.6 & 11.7 & 2.8 & 9.5 & 46.3 & 7.1 & 49.3 & 36.3 & 2.18 \\ \text { erythrophylla } & \pm 3.7 & \pm 1.5 & \pm 2.3 & \pm 0.6 & \pm 2.3 & \pm 2.6 & \pm 2.5 & \pm 3.2 & \pm 2.0 & \\ \text { Neptunia } & 80.3 & 35.6 & 10.8 & 3.5 & 8.0 & 42.1 & 6.3 & 23.0 & 38.1 & 0.87 \\ \text { oleraceae } & \pm 1.5 & \pm 2.5 & \pm 1.4 & \pm 0.1 & \pm 2.2 & \pm 4.5 & \pm 0.7 & \pm 3.1 & \pm 2.6 & \\ \text { Solanum } & 67.6 & 33.7 & 11.6 & 4.7 & 7.1 & 43.2 & 5.9 & 33.2 & 11.2 & 1.25 \\ \text { signum } & \pm 2.4 & \pm 4.0 & \pm 1.1 & \pm 1.0 & \pm 1.6 & \pm 2.7 & \pm 2.5 & \pm 2.4 & \pm 1.3 & \end{array}$

